\title{
Text Documents Classification in Uzbek Language
}

\author{
O.J. Babomuradov Ozod, N. S. Mamatov, L. B. Boboev, B.Otaxonova
}

\begin{abstract}
This article deals with intellectual analyzing technologies, which classify texts in Uzbek language, in which the Bernoulli and multi-nominal models are considered. The textual documents used in this research are from the authentic sources of The State National Information Agency of Uzbekistan. To compare the probability methods of classification, 600 documents of 6 types of categories, with 169205 words, have been used.
\end{abstract}

\section{Keywords: Uzbek Language, classification}

\section{INTRODUCTION}

Text classification is done manually, with the help of expert instructions and machine learning methods [4-6]. Automatic classification of texts is mostly based on the concept of "similarity." Normally, such texts store similar words and word phrases in them.

One of the widespread methods of pre-processing of texts is Bag of Words [1]. In this model, firstly we create vocabulary $\mathrm{V}$ out of the words from the pre-set of texts. A histogram vector is created based on the number of repetitions of the words in the texts that match the vocabulary. Some methods look to shorten the vocabulary [2], and some improve the histogram by using the weight scheme. For example: TF-IDF (term frequency - inverse document frequency) method [1, 3].

In some cases of text classification, based on intellectual information technologies, naive Bayes classifier could be natural language automatically. In order to solve these issues, parameters are normalized.

\section{STATEMENT OF A PROBLEM AND THE CONCEPT OF THE PROBLEM DECISION}

Assume we have a $V$ set of words of a language. Usually $V$ set is called vocabulary. The validity of the $V \quad N(N=|V|)$ is equal to the number of words in it. Based on the $V$ set, a vector of $S=\left(S_{1}, S_{2}, \ldots, S_{N}\right)$ words is formed. The $K=\bigcup_{i=1}^{m} K_{i}$ set of texts, say, is categories.

Revised Version Manuscript Received on 16 September, 2019.

O.J.Babomuradov Ozod1, Tashkent university of information technologies named after Muhammad al-Khwarizmi

N. S. Mamatov2, Scientific and Innovation Center of Information and Communication Technologies at TUIT named after Al-Kharezmi, Tashkent, Uzbekistan

L. B. Boboev3, Scientific and Innovation Center of Information and Communication Technologies at TUIT named after Al-Kharezmi, Tashkent, Uzbekistan

B.Otaxonova4, Tashkent university of information technologies named after Muhammad al-Khwarizmi helpful, but it will be problematic when we try to classify a

* Correspondence Author

Say, we have a $D_{j}$ text of $K_{i}$ category: $(i=\overline{1, m} ; j=\overline{1, p})$. The probability of the $D_{j}$ text lying in the $K_{i}$ set, according to the Bayes theorem $P\left(K_{i} \mid D_{j}\right)$, is equal to:

$$
P\left(K_{i} \mid D_{j}\right)=\frac{P\left(D_{j} \mid K_{i}\right) P\left(K_{i}\right)}{P\left(D_{j}\right)} \Rightarrow P\left(D_{j} \mid K_{i}\right) P\left(K_{i}\right)
$$

With the given $D_{j}$ text, $G_{j}$ set of words is formed and $W_{j}=\left(w_{j 1}, w_{j 2}, \ldots, w_{j r}\right)$ vector of words is created, that matches the $G_{j}$ set.

Based on the vector of $S$ words, $X_{j}^{i}$ Boolean vector, with $N$ dimension, is formed:

$$
x_{j t}=\left\{\begin{array}{l}
1, \quad \text { if } \quad s_{t}=w_{j e} \\
0, \quad \text { otherwise } \quad t=\overline{1, N} ; e=\overline{1, p} ;
\end{array}\right.
$$

If the probability of the $s_{t}$ word is in the $K_{i}$ is $P\left(s_{t} \mid K_{i}\right)$, then the probability of $s_{t}$ is not in $K_{i}$ equals to $\left(1-P\left(s_{t} \mid K_{i}\right)\right)$. Then according to (1), the probability of $D_{j}$ text belongs to $K_{i}$ will be determined thus:

$P\left(D_{j} \mid K_{i}\right)=P\left(S \mid K_{i}\right)=\prod_{t=1}^{|V|}\left[x_{t} P\left(s_{t} \mid K_{i}\right)+\left(1-x_{t}\right)\left(1-P\left(s_{t} \mid K_{i}\right)\right)\right]$ (2) Say the number of documents (that have $s_{t}$ words from $K_{i}$ ) is $\eta_{K_{i}}\left(s_{t}\right)$ and the number of documents that belong to that category is $N_{K_{i}}$; Then the probability of $s_{t}$ word is equal to:

$$
\hat{P}\left(s_{t} \mid K_{i}\right)=\frac{\eta_{K_{i}}\left(s_{t}\right)}{N_{K_{i}}}
$$

If the total number of the learning documents is $N$, then the probability of documents belonging to $K_{i}$ is:

$$
\hat{P}\left(K_{i}\right)=\frac{N_{K_{i}}}{N}
$$

The Bernoulli model of classification of the set of learning documents and the texts of $K_{i}$ category is carried out through the following steps:

1. $V$ vocabulary is created.

2. Learning.

3. Classification

To determine a category of a non-classified document $D$, the combinations (1) and (2) are used:

$$
\begin{aligned}
& P\left(K_{i} \mid S\right) \Rightarrow P\left(S \mid K_{i}\right) P\left(K_{i}\right) \Rightarrow \\
& P\left(K_{i}\right) \prod_{t=1}^{|V|}\left[x_{t} P\left(s_{t} \mid K_{i}\right)+\left(1-x_{t}\right)\left(1-P\left(s_{t} \mid K_{i}\right)\right)\right]
\end{aligned}
$$

In order to classify texts of greater magnitude, usually multi-nominal model is used, which is more effective than the Bernoulli model. Below is a detailed explanation of it.

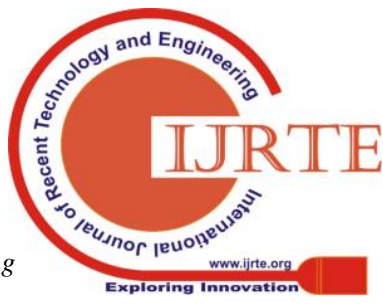


In the multi-nominal model, a vector of signs is created based on the repetition of a word in a vocabulary-based text.

Multi-nominal division comprises the basis of multi-nominal model. Multi-nominal coefficient for $N$ words of $m$ type is calculated with the below formula:

$$
M_{k}=\frac{N !}{n_{1} ! n_{2} ! \ldots n_{2} !}
$$

Here, $n_{i}$ is the amount of repetition $i$ word from the given vocabulary.

Multi-nominal division of words based on the multi-nominal coefficient is calculated with the following formula:

$$
P(N)=\frac{N !}{n_{1} ! n_{2} ! \ldots n_{N} !} p_{1}^{n_{1}} p_{2}^{n_{2}} \ldots p_{N}^{n_{N}}=\frac{N !}{\prod_{t=1}^{N} n_{t} !} \prod_{t=1}^{N} p_{t}^{n_{t}}
$$

Here, the probability of $n_{i}$ words' sequence is divided by the $\prod_{t=1}^{N} p_{t}^{n_{t}}$ multiplication, and classify the target.

Say, $n_{t}$ is the frequency of $s_{t}$ word in a $D_{j}$ document. In that case, the probability of $s_{t}$ is in the $K_{i}$ equals to: $P\left(s_{t} \mid K_{i}\right)$. Then, the probability of $D_{j}$ text belongs to $K_{i}$, i.e. the probability of $S$ words belong to $K_{i}$ is:

$$
P\left(D_{j} \mid K_{i}\right)=P\left(S \mid K_{i}\right)=\frac{N !}{\prod_{t=1}^{|V|} n_{t} !} \prod_{t=1}^{|V|} P\left(s_{t} \mid K_{i}\right)^{n_{t}} \Rightarrow \prod_{t=1}^{|V|} P\left(s_{t} \mid K_{i}\right)^{n_{t}}
$$

Due to the fact that the normalization doesn't concern whether the $s_{t}$ word is the property of any class, it is not necessary to conduct a normalization.

In the multi-nominal model, the probability of the $P\left(s_{t} \mid K_{i}\right)$ category document and $P\left(K_{i}\right)$ category will develop parameters for the model. Whether $D_{j}$ document belongs to $K_{i}$ category, is created by evaluating the parameters of a set of learning documents, and valued with 1 or 0 . When the total number of documents is $N, P\left(s_{t} \mid K_{i}\right)$ probability is determined through the below formula:

$$
\hat{P}\left(s_{t} \mid K_{i}\right)=\frac{\sum_{j=1}^{N} n_{j t} z_{f i}}{\sum_{f=1}^{|V|} \sum_{j=1}^{N} n_{j f} z_{j i}}=\frac{n_{i}\left(s_{t}\right)}{\sum_{f=1}^{|V|} n_{i}\left(s_{f}\right)}
$$

$\left\{Y_{1}, Y_{2}, \ldots, Y_{l}\right\}$ is formed based on the set of learning documents, that is, if $Y_{t}$ belongs to $K_{i}$ category, $z_{t i}$ variable is 1 , otherwise it is 0 .

Say, $Y$ set of learning documents and $K$ set of categories are given, the algorithm of text classification based on multi-nominal model would be as follows:

1. $V$ vocabulary is developed;

2. The followings will be calculated:

$-N-$ total number of documents

- $N_{k}$ - the number of documents, that belong to category $k$, is determined $k=\overline{1, K}$

$-n_{i t}$ the frequency of the word $s_{t}$ in $D_{i}$ document, for each word in $V$, is calculated; simultaneously, the $n_{i}\left(s_{t}\right)$

frequency of $s_{t}$ words in $K_{i}$ category documents is determined;

3. Using (8), $P\left(s_{t} \mid K_{i}\right)$ probability is calculated.

4. Using (4), $P\left(K_{i}\right)$ probability is calculated.

5. Whether a text belongs to $K_{i}$ category is found out thus.

When classifying the $D_{j}$ document, the category probability is calculated through the combinations of (1) and (7):

$$
P\left(K_{i} \mid D_{j}\right)=P\left(K_{i} \mid S\right) \Rightarrow P\left(S \mid K_{i}\right) P\left(K_{i}\right) \Rightarrow P\left(K_{i}\right) \prod_{t=1}^{|v|} P\left(s_{t} \mid K_{i}\right)^{n_{t}}
$$

Unlike the Bernoulli model, in the multi-nominal model, words that don't exist ( $\left.s_{t}=0\right)$ in a document don't affect the probability $\left(p^{0}=1\right)$.

If the words in a document are symbolized as $\mathrm{u}$, the probability is calculated as follows:

$$
P\left(K_{i} \mid D_{j}\right) \Rightarrow P\left(K_{i}\right) \prod_{t=1}^{\operatorname{len}(D)} P\left(u_{t} \mid K_{i}\right)
$$

Here, $u_{t}$ is the $t$-nth word in document $D_{j}$.

In experimental procedure, the change in time of transformation was observed. TfidfVectorizer and HashingVectorizer transformation approaches were used to verify the reliability of results, as shown in Figure 1.

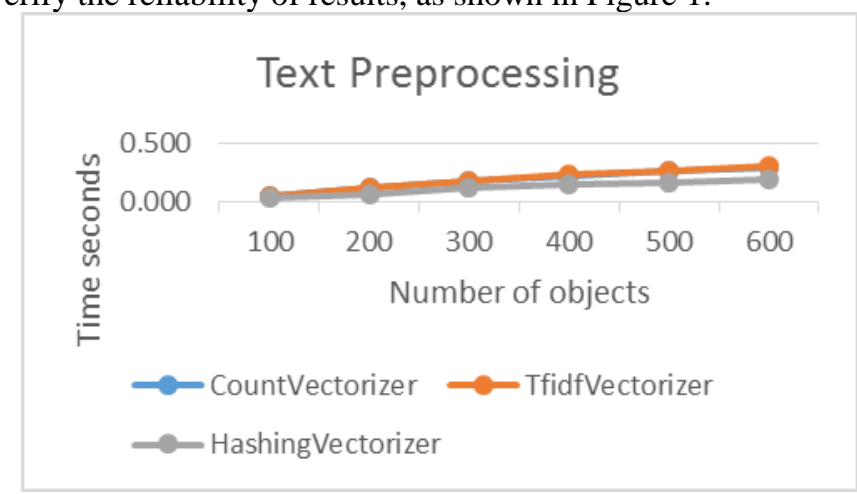

Fig. 1: Time-consuming comparison of different types of transformation

Here are results of algorithm based on Naive Bayes probability with CountVectorizer. Classification accuracy being increased from $78 \%$ to $88 \%$.

When applying TfidfVectorizer approach, speed and accuracy were low: 78-88\% accuracy was obtained.

The following table shows the result of a comparison models' accuracy and time consuming (Table 1).

Table 1: Comparison of classification models

\begin{tabular}{lll}
\multicolumn{1}{c}{ Model } & \multicolumn{1}{c}{ Precision } & \multicolumn{1}{c}{ Time } \\
BernoulliNB & 0.65 & 0.017 \\
MultinomialNB & $\mathbf{0 . 8 6}$ & $\mathbf{0 . 0 0 9}$ \\
LinearSVC & 0.82 & 0.89 \\
Perceptron & 0.86 & 0.019 \\
\hline
\end{tabular}

To assess the effectiveness of the classification models such as the Bernoulli and multi-nominal, 600 documents, with 169205 words of 6 categories in it, have been used and with the set of documents, a 28343-word vocabulary has been created. 
When testing the classification of the selected texts with the Bernoulli model, average accuracy was $65 \%$ and it took 17.28 milliseconds. As for the multi-nominal model, the accuracy came to about $86 \%$ and it took 9.79 milliseconds. Experimental research works have proven the multi-nominal model more accurate and faster than the Bernoulli.

\section{CONCLUSION}

Pre-processing of texts, with Bernoulli and multi-nominal methods, has been looked through. The space for symbols, which is the most important for the classification of texts in Uzbek language, and mathematical way of classification have been developed. In order to make them recognizable, texts of various themes were formed and classified into categories. The results show the effectiveness of the multi-nominal model, when classifying the texts of bigger size.

\section{REFERENCES}

1. C.D. Manning, P. Raghavan, H. Schütze. "Introduction to Information Retrieval", Cambridge University Press, Cambridge (2008)

2. R.E.Madsen, S.Sigurdsson, L.K.Hansen, J.Larsen. "Pruning the vocabulary for better context recognition", Proceedings of the International Conference on Pattern Recognition, 2 (2004), pp. 483-488

3. J.H. Paik. "A novel tf-idf weighting scheme for effective ranking", Proceedings of the International ACM SIGIR Conference on Research and Development in Information Retrieval (2013), pp. 343-352

4. S.B. Kim, K.S. Han, H.C. Rim, S.H. Myaeng. "Some Effective Techniques for Naive Bayes Text Classification", IEEE Transactions on Knowledge and Data Engineering, December 2006

5. Y.Yiming, J.Thorsten "Text categorization", (2008), Scholarpedia, 3(5):4242

6. P.Philipp, W.Bonnie "Stable classification of text genres" (2011) Association for Computational Linguistics Vol. 37, No 2., pp.385-393,

7. B.S.Harish, D.S.Guru, S.Manjunath "Representation and classification of text documents: A Brief Review", IJCA Special Issue on "Recent Trends in Image Processing and Pattern Recognition" RTIPPR, 2010., pp.110-119

8. N.Kamal, K.Andrew, T.Sebastian, M.Tom "Text classification from labeled and unlabeled documents using EM", Machine Learning, 1-34 Kluwer Academic Publishers, Boston.

9. A.A.Alekseev, A.S.Katusyev, A.E.Kirillov, A.P.Kirpichnikov "Classification of text documents based on text mining technology" Computer Science, Computer Science and Management, Bulletin of the Technical University. 2016. T.19, No.18, pp.116-119

10. I.V.Polyakov, T.V.Sokolova, A.A.Chepovskii, A.M.Chepovsky "The problem of classification of texts and differentiating signs" Bulletin of the Novosibirsk State University of shock. Series: Information technology. 2015. Vol. 13, No. 2., pp. 55-63 